\title{
ZEOLITAS EN LA FERTILIZACIÓN QUÍMICA DEL CACAO CCN-51 ASOCIADO CON CUATRO ESPECIES MADERABLES
}

\author{
ZEOLITES CHEMICAL FERTILIZATION OF CACAO CCN-51 ASSOCIATED \\ WITH FOUR TIMBER SPECIES
}

\author{
Fernando Sánchez-Mora ${ }^{1}$, Gregorio Vásconez Montúfar ${ }^{1,2}$, Fabián Abril Saltos ${ }^{2}$, Jaime Vera Chang ${ }^{1,2}$, \\ Rommel Ramos Remache ${ }^{1,2}$, Gorki Díaz Coronel ${ }^{1,2}$, Emma Torres Navarrete ${ }^{2}$, Germán Jácome López ${ }^{2}$ \\ ${ }^{1}$ Dirección de Investigación Cientifica y Tecnológica, Universidad Técnica Estatal de Quevedo. Campus Finca Experimental \\ "La María", km 7 vía Quevedo-El Empalme. C. P. 73. Mocache, Los Ríos, Ecuador. \\ ${ }^{2}$ Facultad de Ciencias Pecuarias, Universidad Técnica Estatal de Quevedo. Campus Finca Experimental "La María", \\ km 7 vía Quevedo-El Empalme. C. P. 73. Mocache, Los Ríos, Ecuador.
}

Emails: fernandosanchezm23@hotmail.com; goyitouteq@hotmail.com; fabianabril80@yahoo.com; jverac@uteq.edu.ec; rramos@uteq.edu.ec; gorky_diaz@hotmail.com; emdatona@hotmail.com; gercurry@hotmail.com

\section{RESUMEN}

$\mathrm{S}$ e evaluó el efecto de dos zeolitas comerciales del tipo clinoptilolita: Roca Mágica ${ }^{\circledR}$ (RM) y Zeolite $\mathrm{C}^{\circledR}(\mathrm{ZC})$, incluidas en la fertilización química sobre la producción y rentabilidad del cacao "CCN-51", de origen sexual, asociado con cuatro especies maderables [Caoba de montaña (Colubrina arborescens (Mill.) Sarg), fernánsanchez (Triplaris cumingiana F.), guayacán blanco (Cybistax donell-smithii Rose) y laurel prieto (Cordia macrantha Chadat)]. En la fertilización fueron utilizados los dos tipos de zeolita (RM y ZC) y dos niveles (25 y 30\%), más un testigo (sin zeolita). La fertilización se fraccionó en tres partes aplicándose urea, $\mathrm{MgSO}_{4}, \mathrm{~K}_{2} \mathrm{O}$ y $\mathrm{P}_{2} \mathrm{O}_{5}$. Se empleó un diseño de parcelas divididas con cuatro especies maderables, cinco subtratamientos (tipos y niveles de zeolita) y un testigo, en cuatro repeticiones. Se utilizó la prueba de Tukey al 5\% de probabilidad. Se midió la fenología del cacao (brotación, floración, fructificación), cherelles wilt, número de mazorcas sanas y producción de cacao en $\mathrm{kg} \mathrm{ha}^{-1}$. El mayor número de brotes los emitieron las plantas de cacao asociadas con fernánsanchez en la época lluviosa, posiblemente por la menor cobertura aérea de esta especie. El cacao alcanzó su mayor fructificación en la época lluviosa con las asociaciones fernánsanchez y guayacán y, 30\% de ZC. El mayor número de mazorcas sanas, y producción de cacao fue obtenido con la asociación fernánsanchez en la época seca. La mayor rentabilidad se encontró con fernánsanchez y $25 \%$ de RM.

Palabras clave: Producción de cacao, sistemas agroforestales, fertilización, zeolitas.

Recibido: 10-enero-2013. Recibido en forma corregida: 7-marzo-2013.

Aceptado: 29-mayo-2013.

Publicado como ARTÍCULO CIENTÍFICO en Ciencia y Tecnología 6(2): 21-29

Julio-Diciembre de 2013

ISSN 1390-4051 impreso; ISSN 1390-4043 electrónico

(C) Ciencia y Tecnología. UTEQ. Quevedo-Ecuador
$\mathrm{T}$ he effect of two commercial zeolites of the clinoptilolite type was evaluated: Roca Magica ${ }^{\circledR}$ (RM) and Zeolite $\mathrm{C}^{\circledR}(\mathrm{ZC})$, included in the chemical fertilization on the production and profitability of cocoa "CCN-51" of sexual origin, associated with four timber species, caoba de montaña (Colubrina arborescens (Mill.) Sarg), fernánsanchez (Triplaris cumingiana F.), guayacán blanco (Cybistax donell-smithii Rose) and laurel prieto (Cordia macrantha Chadat). For fertilization two different levels (25 and 30\%) of two types of zeolite (RM and ZC) were used. Fertilization experiments were divided into three parts, applying urea, $\mathrm{MgSO}_{4}, \mathrm{~K}_{2} \mathrm{O}$ and $\mathrm{P}_{2} \mathrm{O}_{5}$. A split plot design was used with four timber species, five subtreatments (types and levels of zeolite) and four replications. Cocoa phenology (sprouting, flowering, fruiting) as well as cherelles wilt, healthy pods number and cocoa production in $\mathrm{kg} \mathrm{ha}^{-1}$ was measured. Cocoa plants associated with fernánsanchez issued the highest number of outbreaks in the rainy season, probably due to the lower air coverage of the species. The highest fructification of cacao was achieved in the rainy season with fernánsanchez and guayacán associations, applying $30 \%$ of ZC. The highest number of healthy pods, and cocoa production was obtained with fernánsanchez association in the dry season, while the highist profitability was found with fernánsanchez and $25 \%$ of RM.

Key words: Cocoa production, agroforestry systems, fertilization, zeolites. 


\section{INTRODUCCIÓN}

$\mathrm{L}$ a agroforestería tiene el potencial para resolver o aliviar muchos de los problemas agrícolas de manera ecológica (Wojtkowski et al., 1991). Los esfuerzos de conservación promueven diversos usos de la tierra y sistemas de cultivo, entre los que se destacan diversos sistemas agroforestales como alternativa al monocultivo (Dahlquist et al., 2007). La aplicación de técnicas agroforestales puede consolidar o aumentar la productividad de establecimientos agropecuarios y plantaciones forestales de diversas dimensiones o, por lo menos evitar que haya degradación del suelo o merma de la productividad en el curso de los años (MusálemSantiago, 2002), reduciendo inclusive el estrés en las plantas (Beer et al., 1998). Por otro lado, los sistemas agroforestales constituyen una estrategia que permiten a los cultivos enfrentar eventos adversos como sequías extensas o heladas (Schwendenmann et al., 2010).

En la naturaleza existen asociaciones esenciales entre especies vegetales que favorecen su crecimiento, desarrollo y producción. Las plantas umbrofilas como el cacao (Theobroma cacao L.), cultivo de interés en muchas zonas tropicales, presentan especial actitud a la asociación con especies vegetales capaces de proporcionarle sombra. Desde esta perspectiva, la asociación del cultivo de cacao con especies maderables de rápido crecimiento contribuye a los requerimientos de sombra del cultivo, y permite la diversificación de los ingresos al productor (cacao a corto plazo y madera a largo plazo).

El cacao es uno de los rubros agrícolas más importantes para el Ecuador, cubriendo el 5\% de la producción mundial, siendo uno de los cultivos tradicionales de interés comercial en la provincia de Los Ríos (Sánchez-Mora et al., 2011). Durante los primeros dos años de edad del cacaotal se recomiendan proyecciones de sombra del 60\% (Jaimez et al., 2008) y en plantaciones adultas es necesario que el sombramiento se mantenga entre el 25 y 30\% (Enríquez, 2004). Se debe considerar especies forestales establecidas en la zona, con un comportamiento apropiado para proporcionar sombra a las plantas de cacao, arquitectura del tallo erecto y copa poco densa (Palencia et al., 2006). Es preciso encontrar alternativas de compensación económica para los productores de cacao para incentivar la conservación de especies maderables nativas en las plantaciones (Sambuichi, 2006). Incluyen cultivos cosechados regularmente como el banano o plátano que proporciona ingresos a corto plazo, además de la renta temporal generada a partir de cacao (Dahlquist et al., 2007).

Los sistemas agroforestales de cacao asociados con especies forestales maderables, frutales e industriales son los sistemas que presentan mayor eficiencia en la fijación de carbono comparado a los sistemas tradicionales de uso de la tierra (Concha et al., 2007). Se puede integrar la producción sostenible y la fijación de carbono en las fincas cacaoteras mediante el manejo y diversificación de los cacaotales, evitando el cambio de uso del suelo y reforestando las fincas con especies frutales y maderables (Orozco et al., 2008).

En los trópicos se encuentran diferentes combinaciones de sistemas agroforestales que varían de acuerdo a la región, posibilidades económicas y factibilidad de mercado de los componentes del sistema, en la mayoría de las nuevas combinaciones agroforestales el manejo se ha realizado tomando como ejemplo experiencias de los sistemas agroforestales tradicionales, incluyendo la aplicación de fertilizantes (Souza y Dias, 2005; citado por Jaimez et al., 2008). En muchos casos, por una aproximación dominantemente empírica, se desconoce si los aportes nutricionales a través del fertilizante suplen lo faltante, o si, al contrario existe sobredosis y gastos innecesarios (Barber, 1995). En el caso de experiencias con cacao las evaluaciones con diferentes tipos de fertilización ha dado resultados que varían en función del tipo de suelo, fertilizante, cultivo y en diferentes momentos de disponibilidad de agua, entre otros (Souza y Dias, 2005; citado por Jaimez et al., 2008).

Por otro lado, las zeolitas son aluminosilicatos cristalinos que presentan una estructura tridimensional (Ramesh y Reddy, 2011), a las cuales se les atribuyen numerosas propiedades como su capacidad para perder y ganar agua reversiblemente y ser capaces de intercambiar selectivamente una variedad de cationes sin presentar cambios mayores en su estructura básica (Collazos-García, 2010). Las características físico-químico de las zeolitas constituyen un recurso importante de gran aplicación en la agricultura, así como en una alternativa muy atractiva, por su contribución a la reducción de la pérdida de nitrógeno en más de un 30\%, la disminución de los niveles de fertilizante sin afectar los rendimientos, la calidad de los cultivos, así como la reducción en la contaminación ambiental (Jhon et al., 1998). Las zeolitas más usadas en la agricultura son la clinoptilolita y la modernita, que al entrar en contacto con el amonio, lo retienen en su estructura interna y externa, funcionando entonces como un fertilizante nitrogenado de liberación lenta (Flores et al., 2007).

Sobre esta base la presente investigación se desarrolló orientando sus acciones hacia un mejor aprovechamiento de los nutrientes que se aplican al suelo, particularmente del nitrógeno. La zeolita como aditivo en la fertilización, es una alternativa tecnológica 
interesante a ser estudiada, por lo que el objetivo de este trabajo fue establecer el tipo y la dosis de zeolita que mejore la producción del cacao "CCN-51", de origen sexual, asociado a cuatro especies maderables.

\section{Materiales y métodos}

$L^{a}$ investigación se ejecutó en la Finca Experimental “La Represa”, propiedad de la Universidad Técnica Estatal de Quevedo (UTEQ), Quevedo, provincia de Los Ríos, cuyas coordenadas geográficas son $79^{\circ} 30^{\prime} 23^{\prime \prime}$ de longitud oeste y $01^{\circ} 00^{\prime} 35^{\prime \prime}$ de latitud sur, ubicada en la parte alta de la cuenca del Río Guayas a $90 \mathrm{msnm}$. La fase de la investigación tuvo una duración de 12 meses, durante el año 2007.

\section{Requerimientos nutricionales y fertilización}

Para una eficiente fertilización se realizó un análisis del suelo, donde se encontró que el suelo era rico en potasio, con deficiencias de nitrógeno y fósforo, con un alto contenido de materia orgánica y textura franco arcillosa. Como fuente de nitrógeno, fósforo, potasio, magnesio y azufre se utilizó: urea $(46 \% \mathrm{~N})$, súper fosfato triple $\left(46 \% \mathrm{P}_{2} \mathrm{O}_{5}\right)$, muriato de potasio $\left(60 \% \mathrm{~K}_{2} \mathrm{O}\right)$ y sulfato de magnesio $\left(46 \% \mathrm{MgSO}_{4}\right)$ más la inclusión de las zeolitas "Roca mágica ${ }^{\mathbb{} "}$ " $(\mathrm{RM})$ y "Zeolite $\mathrm{C}^{\mathbb{}}$ " $(\mathrm{ZC})$, en porcentajes de 25 y $30 \%$ del peso total de fertilizante aplicado por árbol. Al tratamiento testigo se le aplicó la misma cantidad de fertilizantes a excepción de zeolita.

La fertilización se fraccionó en tres partes: la primera fertilización se realizó en el mes de abril/2006 con una aplicación por árbol de cacao de $123 \mathrm{~g}$ de urea, $75 \mathrm{~g} \mathrm{MgSO}_{4}, 168 \mathrm{~g} \mathrm{~K}_{2} \mathrm{O}, 50.5 \mathrm{~g} \mathrm{P}_{2} \mathrm{O}_{5}$; la segunda aplicación fue realizada en el mes de julio/2006 con una aplicación de $123 \mathrm{~g}$ de urea, $75 \mathrm{~g} \mathrm{MgSO}_{4}$ y la última aplicación fue realizada en el mes de octubre/2006 con una aplicación de $123 \mathrm{~g}$ de urea, $75 \mathrm{~g} \mathrm{MgSO}_{4}$ y durante la época seca se realizó después de un riego.

\section{Material genético}

El material genético empleado fue el hibrido de cacao "CCN-51" proveniente de semilla de 11 años de edad, sembrados a una distancia de 3 x 3 m (988 árboles ha $\left.^{-1}\right)$. Y las especies maderables: Caoba de montaña (Colubrina arborescens (Mill.) Sarg), fernánsanchez (Triplaris cumingiana F.), guayacán blanco (Cybistax donell-smithii Rose) y laurel prieto (Cordia macrantha Chadat) de 12 años de edad y sembradas a una distancia de 9 × $9 \mathrm{~m}$ (123 árboles ha-1).

\section{Factores, niveles y variables evaluadas}

Se estudió en el cacao "CCN-51" de propagación sexual, el efecto de dos tipos de zeolita (RM y ZC) y dos niveles ( 25 y $30 \%$ ) y un testigo, en la asociación de cuatro especies maderables (guayacán, laurel, fernánsanchez y caoba).

Las variables fenológicas brotación, floración, fructificación y la variable fisiológica cherelles wilt, fueron registradas mensualmente empleando una escala arbitraria de 0 a 4 dependiendo del grado de emisión de brotes, flores, frutos y cherrelles de las plantas en cada unidad experimental. Donde $0=0 \%$ (nula); $1=$ $25 \%$ (poca); $2=50 \%$ (ligera); $3=75 \%$ (moderada) y $4=$ $100 \%$ (abundante), posteriormente estos datos fueron expresados por época.

El número de mazorcas sanas, se removió mensualmente los frutos sanos y maduros, libres de enfermedades, registrados al momento de la cosecha.

El rendimiento de cacao seco por año $\left(\mathrm{kg} \mathrm{ha}^{-1}\right)$, se estimó con el número de mazorcas sanas cosechadas durante un año dividiendo para el índice de mazorca en cada subparcela. Este resultado se transformó a hectárea y se obtuvo el rendimiento de cacao seco en $\mathrm{kg}$.

\section{Diseño experimental y análisis estadístico}

Se empleó un diseño de parcelas divididas con cuatro tratamientos (especies maderables) y cinco subtratamientos (tipos y niveles de zeolita) y cuatro repeticiones. El experimento fue establecido en 10,000 $\mathrm{m}^{2}$, en cada parcela útil fueron evaluadas 20 plantas de cacao y nueve árboles maderables.

El modelo estadístico es:

$X i j k=\mu+\rho i+\alpha j+\delta i j+\beta k+(\alpha \beta) j k+\epsilon i j k$.

Dónde:

$\mu=$ Media de observación

$\rho \mathrm{i}=$ Efecto de bloque

$\alpha j=$ Efecto de la especies maderables

$\delta \mathrm{ij}=$ Componente al azar de la parcela grande

$\beta \mathrm{k}=$ Efecto de los tipos y niveles de zeolita

$(\alpha \beta) \mathrm{jk}=$ Interacción de las especies maderables con los tipos y niveles de zeolita

$\epsilon \mathrm{ijk}=$ Error experimental.

Para la comparación entre las medias de tratamientos y subtratamientos se utilizó la prueba de Tukey al 5\% de probabilidades. Además el estudio de los grupos de niveles de zeolita se realizó con el uso de polinomios ortogonales.

\section{Tratamientos}

De la combinación de los niveles especies maderables (guayacán, laurel, fernánsanchez y caoba), tipos de zeolita (RM y ZC) y dosis de zeolita (25 y $30 \%$ ), más un testigo (fertilización sin zeolita) resultaron 20 tratamientos (Cuadro 1). 
Cuadro 1. Tratamientos producto de la combinación de las especies maderables y los tipos y dosis de zeolita

\begin{tabular}{cllc}
\hline No $^{\circ}$ & Especie maderables & \multicolumn{1}{c}{$\begin{array}{c}\text { Tipos de } \\
\text { zeolita }\end{array}$} & Dosis (\%) \\
\hline 1 & Guayacán blanco & - & Sin zeolita \\
2 & Guayacán blanco & Roca Mágica & 25 \\
3 & Guayacán blanco & Roca Mágica & 30 \\
4 & Guayacán blanco & Zeolite C & 25 \\
5 & Guayacán blanco & Zeolite C & 30 \\
6 & Laurel prieto & - & Sin zeolita \\
7 & Laurel prieto & Roca Mágica & 25 \\
8 & Laurel prieto & Roca Mágica & 30 \\
9 & Laurel prieto & Zeolite C & 25 \\
10 & Laurel prieto & Zeolite C & 30 \\
11 & Fernánsanchez & - & Sin zeolita \\
12 & Fernánsanchez & Roca Mágica & 25 \\
13 & Fernánsanchez & Roca Mágica & 30 \\
14 & Fernánsanchez & Zeolite C & 25 \\
15 & Fernán Sánchez & Zeolite C & 30 \\
16 & Caoba de montaña & - & Sin zeolita \\
17 & Caoba de montaña & Roca Mágica & 25 \\
18 & Caoba de montaña & Roca Mágica & 30 \\
19 & Caoba de montaña & Zeolite C & 25 \\
20 & Caoba de montaña & Zeolite C & 30 \\
\hline
\end{tabular}

\section{RESULTADOS Y DISCUSIÓN}

Zn la emisión de brotes de las plantas de cacao - al utilizar diferentes especies maderables se presentaron diferencias significativas en la época lluviosa $(\mathrm{p}<0.05)$, en la época seca no hubieron diferencias. Con respecto al efecto de los tipos y niveles de zeolita, no se reportaron diferencias (Cuadro 2). Con la asociación fernánsanchez se registró los valores mayores de brotación en la época lluviosa, resultado que posiblemente se debió a la menor cobertura aérea presente de esta especie, que permitió un mayor ingreso de luz solar a las plantas de cacao, lo que contribuyó a una mayor actividad fotosintética emitiendo una mayor cantidad de brotes. Esto concuerda con Braudeau (1981), quien señala que es muy importante la intensidad de las radiaciones solares y sus efectos térmicos sobre ciertos fenómenos fisiológicos, tales como el desarrollo de las brotaduras foliares y el crecimiento del cacao. Así mismo, se ha demostrado una relación directa entre la fotosíntesis y la disponibilidad de carbohidratos solubles en las plantas (Zhu et al., 2008), los mismos que son útiles en la formación de nuevas estructuras vegetales (brotes vegetativos y reproductivos) (Scholefield et al., 1985; Zhu et al., 2008).

La floración del cacao fue superior con las asociaciones laurel, fernánsanchez y guayacán en relación a la especie maderable caoba, en las épocas seca y lluviosa $(\mathrm{p}<0.05)$, Con respecto al efecto de los tipos y niveles de zeolita, no se reportaron diferencias

Cuadro 2. Efecto de cuatro especies maderables y dos tipos y niveles de zeolita incluidas en la fertilización química sobre la brotación, floración y cherelles wilt del cacao "CCN-51" de semilla, en dos épocas del año

\begin{tabular}{lcccccc}
\hline & \multicolumn{2}{c}{ Brotación $^{1 /}$} & \multicolumn{2}{c}{ Floración $^{1 /}$} & \multicolumn{2}{c}{ Cherelles wilt $^{1 /}$} \\
\cline { 2 - 7 } Factores/niveles & \multicolumn{7}{c}{ Épocas } \\
\cline { 2 - 8 } & \multicolumn{7}{c}{ Seca } & Lluviosa & Seca & Lluviosa & Seca & Lluviosa \\
\hline Especies maderables & & & & & \\
Guayacán & 0.51 & $0.63 \mathrm{~b}$ & $1.13 \mathrm{ab}$ & $1.22 \mathrm{~b}$ & 0.63 & $0.73 \mathrm{ab}$ \\
Laurel & 0.47 & $0.71 \mathrm{ab}$ & $1.18 \mathrm{a}$ & $1.39 \mathrm{a}$ & 0.68 & $0.65 \mathrm{ab}$ \\
Fernánsanchez & 0.47 & $0.86 \mathrm{a}$ & $1.12 \mathrm{ab}$ & $1.28 \mathrm{ab}$ & 0.73 & $0.81 \mathrm{a}$ \\
Caoba & 0.37 & $0.81 \mathrm{ab}$ & $0.92 \mathrm{~b}$ & $1.18 \mathrm{~b}$ & 0.66 & $0.57 \mathrm{~b}$ \\
Tipos y niveles de zeolita & & & & & & \\
Sin Zeolita & 0.45 & 0.71 & 1.10 & 1.25 & 0.63 & 0.61 \\
Roca Mágica 25\% & 0.44 & 0.76 & 1.09 & 1.24 & 0.75 & 0.68 \\
Roca Mágica 30\% & 0.47 & 0.76 & 1.12 & 1.28 & 0.69 & 0.71 \\
Zeolite C 25\% & 0.43 & 0.77 & 1.04 & 1.29 & 0.69 & 0.75 \\
Zeolite C 30\% & 0.48 & 0.76 & 1.10 & 1.27 & 0.60 & 0.70 \\
\hline Error estándar & 0.02 & 0.02 & 0.02 & 0.02 & 0.03 & 0.02 \\
CV (\%) & 23.32 & 15.88 & 17.36 & 12.77 & 30.56 & 23.19 \\
\hline
\end{tabular}

Medias seguidas por la misma letra no presentan diferencias estadísticas (Tukey, $\mathrm{p}>0.05$ ).

1/ Escala: 0 = nula; 1 = poca; 2 = ligera; 3 = moderada; 4 = abundante 
significativas (Cuadro 2). La superioridad del laurel y fernánsanchez en cuanto a la floración, se asocia a la mayor luminosidad que estas especies maderables dejan penetrar debido a su copa más pequeña y estrecha.

La mayor presencia cherelles wilt se registró en la época lluviosa con la especie maderable fernánsanchez $(p<0.05)$, en la época seca no se reportaron diferencias significativas. Con respecto al efecto de los tipos y niveles de zeolita, no se reportaron diferencias (Cuadro 2). Este resultado podría estar relacionado con la mayor fructificación que registró esta especie. La alta fructificación del cacao con el fernánsanchez muestra que este sistema es compatible, y que está muy relacionado con la regulación fisiológica de la planta que determina cuantos frutos puede mantener hasta llegar a la producción, lo que se expresó con la incidencia de cherelles wilt.
La interacción "Especies maderables por tipos y niveles de zeolita" fue significativa en la emisión de brotes de las plantas de cacao en la época de seca $(\mathrm{p}<0.05)$. Durante la época seca, se observó que el cultivo de cacao bajo el guayacán tiene una mayor brotación seguido del fernánsanchez con el $30 \%$ de ZC y $30 \%$ de RM, respectivamente (Figura 1).

El análisis de la interacción "Especies maderables y los tipos y niveles de zeolita" sobre la fructificación del "CCN-51" en el periodo de lluvias, indica que el cultivo de cacao bajo el fernánsanchez alcanza una mayor fructificación con el $30 \%$ de ZC e igual al guayacán; en cambio en el laurel fue con el 30\% de RM (Figura 2). Lo anterior, indica un efecto del cacao asociado al maderable y al tipo de zeolita.

En el análisis de las mazorcas sanas de cacao se encontraron diferencias en la época seca, con las

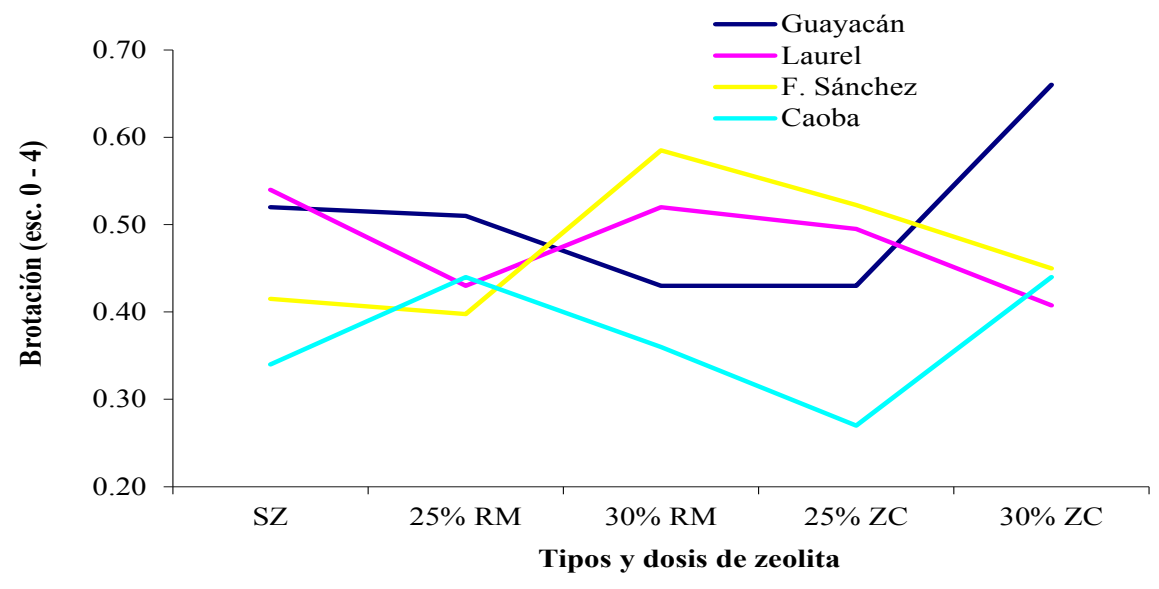

Figura 1. Interacción "Especie maderable por tipos y niveles de zeolita" en la brotación del cacao "CCN-51" de semilla, en la época seca

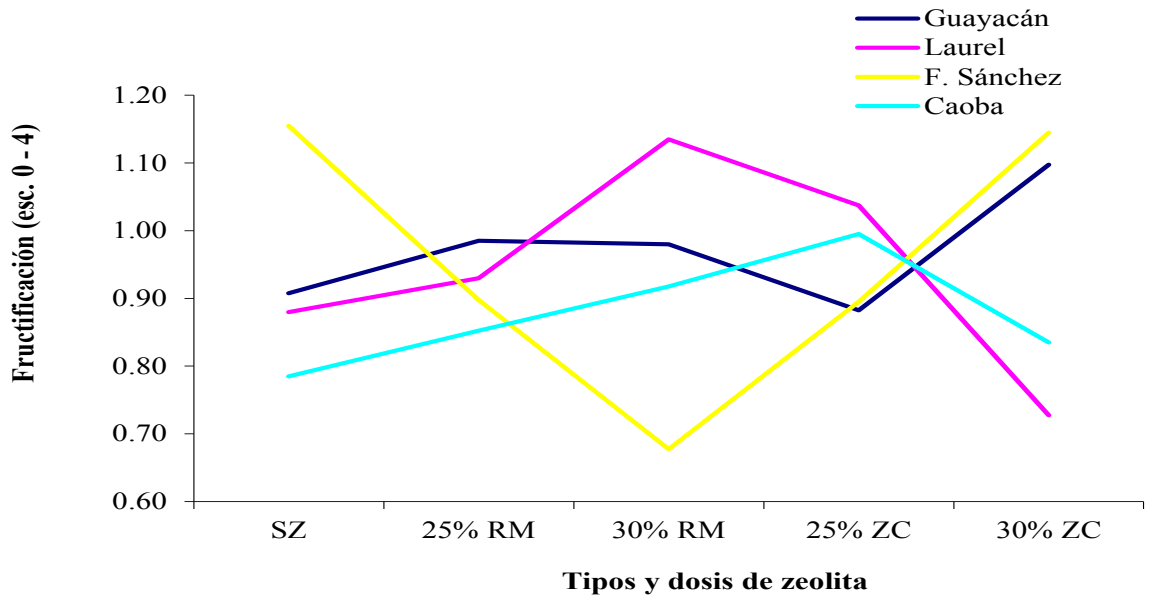

Figura 2. Interacción "Especie maderables por tipos y niveles de zeolita" en la fructificación del cacao "CCN-51" de semilla, en la época lluviosa 
especies maderables $(\mathrm{p}<0.05)$, en la época lluviosa no se reportaron diferencias. Con respecto al efecto de los tipos y niveles de zeolita, no hubo diferencias (Cuadro 3). Los árboles de cacao bajo la influencia de fernánsanchez registraron un mayor número de mazorcas en la época seca. Este comportamiento se atribuye a su alta producción de hojarasca y follaje durante todo el año, lo que ayuda a mantener un mayor contenido de humedad en el suelo; además, el adecuado porcentaje de sombra que proyecta esta especie (25 a 30\%). Lo que concuerda con Jaimez y Franco (1999), que estimaron que la transferencia de nutrientes cubre el $40 \%$ de los requerimientos del cultivo de cacao porcelana en la región de Tucaní-Venezuela bajo la sombra de frutales.

En la producción de cacao, el análisis de varianza mostró diferencias en la época seca, al utilizar diferentes especies maderables $(p<0.05)$, mientras que en la época lluviosa no hubo diferencias. Con respecto al efecto de los tipos y niveles de zeolita, tampoco hubo diferencias significativas (Cuadro 3). Con fernánsanchez se obtuvieron las mayores producciones de cacao (794 $\mathrm{kg} \mathrm{ha}^{-1}$ ), este rendimiento podría estar relacionado con la densidad de siembra puesto que en sistemas agroforestales se tiene menor cantidad de árboles, sin dejar de lado que las plantas utilizadas en este sistema fueron de propagación sexual. Además, se observó que el "CCN-51" en la época seca, presenta mayor producción que en la época lluviosa.

Estos sistemas agroforestales ofrecen al agricultor alternativas de corto, mediano y largo plazo, además de contribuir para el equilibrio del medio ambiente. Isaac et al. (2007) manifiestan que una relación beneficiosa entre los árboles de sombra y biomasa de cacao mejora presumiblemente las capacidades de los ciclos de

Cuadro 3. Efecto de cuatro especies maderables y dos tipos y niveles de zeolita incluidas en la fertilización química sobre el número de mazorcas sanas y el rendimiento de cacao seco (kg ha $\left.{ }^{-1}\right)$ "CCN-51" de semilla en dos épocas del año

\begin{tabular}{lcccc}
\hline & \multicolumn{3}{c}{ Mazorcas sanas } & \multicolumn{2}{c}{ Rendimiento kg ha $^{-1}$} \\
\cline { 2 - 5 } Factores/niveles & \multicolumn{5}{c}{ Elpocas } \\
\cline { 2 - 5 } & Seca & Lluviosa & Seca & Lluviosa \\
\cline { 2 - 5 } Especies maderables & $7.40 \mathrm{~b}$ & 3.35 & $380.71 \mathrm{~b}$ & 166.74 \\
Guayacán & $8.00 \mathrm{ab}$ & 2.85 & $415.09 \mathrm{ab}$ & 150.52 \\
Laurel & $12.00 \mathrm{a}$ & 3.25 & $623.37 \mathrm{a}$ & 170.58 \\
Fernánsánchez & $8.25 \mathrm{ab}$ & 2.85 & $427.39 \mathrm{ab}$ & 144.83 \\
Caoba & & & & \\
Tipos y niveles de zeolita & 7.68 & 3.06 & 385.59 & 161.21 \\
Sin Zeolita & 10.18 & 3.25 & 564.71 & 178.70 \\
Roca Mágica 25\% & 10.06 & 3.18 & 563.71 & 155.67 \\
Roca Mágica 30\% & 7.88 & 2.81 & 397.20 & 143.46 \\
Zeolite C 25\% & 8.63 & 3.06 & 397.01 & 151.79 \\
Zeolite C 30 \% & 0.50 & 0.20 & 28.32 & 10.84 \\
\hline Error estándar & 44.30 & 50.84 & 49.36 & 54.61 \\
CV (\%) & &
\end{tabular}

Medias seguidas por la misma letra no presentan diferencias estadísticas (Tukey, $\mathrm{p}>0.05$ )

Cuadro 4. Contrastes ortogonales del efecto de tipos de zeolita en el número de mazorcas sanas y el rendimiento de cacao seco (kg ha-1) "CCN-51" de semilla asociado con cuatro especies maderables

\begin{tabular}{|c|c|c|c|c|}
\hline \multirow{2}{*}{ Contraste } & \multicolumn{2}{|c|}{ Mazorcas sanas } & \multicolumn{2}{|c|}{ Rendimiento $\mathrm{kg} \mathrm{ha}^{-1}$} \\
\hline & Época seca & Época lluviosa & Época seca & Época lluviosa \\
\hline $\begin{array}{c}\text { Roca Mágica } \\
\text { Vs }\end{array}$ & $9.95 \mathrm{a}$ & $3.16 \mathrm{a}$ & $564.21 \mathrm{a}$ & $167.18 \mathrm{a}$ \\
\hline Zeolite C & $8.14 \mathrm{~b}$ & $2.91 \mathrm{a}$ & $397.10 \mathrm{~b}$ & $147.63 \mathrm{a}$ \\
\hline $\mathrm{CV}(\%)$ & 21.74 & 22.54 & 49.36 & 54.61 \\
\hline
\end{tabular}


nutrientes sugiriendo un potencial de productividad sostenida.

El contraste ortogonal "RM vs ZC" en la producción de mazorcas sanas y producción de cacao presentó diferencias significativas en la época seca. Con RM se encontró un mayor número de mazorcas sanas y rendimiento de cacao seco (Cuadro 4). Se atribuye este mejor comportamiento a sus características físico químicas $\mathrm{y}$, a su estructura cavernosa que actúa como un almacén de nutrientes que los aísla de las fuerzas de retención del suelo e inmovilización de los microorganismos. La aplicación de zeolita en conjunto con la fertilización edáfica, origina un incremento en el rendimiento por planta y por hectárea en comparación con el testigo, en el que solo se utilizó fertilización convencional (Díaz-Coronel et al., 2009).

Los mayores ingresos brutos, ingresos netos y relación beneficio/costo los presentó el fernánsanchez con RM 25\% con 2,033.87 USD; 1,191.12 USD y 1.41, respectivamente (Cuadro 5). Estos resultados se deben a la mayor producción de mazorcas presentada por este tratamiento, lo que influyó en mejores utilidades, lo cual concuerda con Díaz-Coronel et al. (2009), en un análisis económico basado en el rendimiento de la variedad de fréjol "EVG-6", donde la mayor producción y rentabilidad por hectárea, se alcanzó con RM $(25 \%)$ y ZC (75\%).

Cuadro 5. Análisis económico del efecto de cuatro especies maderables, dos tipos y niveles de zeolita incluida en la fertilización química sobre el cacao "CCN-51" de semilla en dos épocas del año

\begin{tabular}{|c|c|c|c|c|c|c|}
\hline Especies maderables & $\begin{array}{c}\text { Tipos y niveles de } \\
\text { zeolita }\end{array}$ & $\begin{array}{l}\text { Costos } \\
\text { totales } \\
\text { (USD) }\end{array}$ & $\begin{array}{l}\text { Producción } \\
\left(\mathrm{kg} \mathrm{ha}^{-1}\right)\end{array}$ & $\begin{array}{c}\text { Ingreso } \\
\text { bruto } \\
\text { (USD) }\end{array}$ & $\begin{array}{c}\text { Ingreso } \\
\text { neto } \\
\text { (USD) }\end{array}$ & $\begin{array}{c}\text { Relación } \\
\mathrm{B} / \mathrm{C}\end{array}$ \\
\hline \multirow{5}{*}{ Guayacán } & Sin Zeolita & 736.73 & 387.12 & 725.23 & -11.50 & -0.02 \\
\hline & Roca Mágica 25\% & 792.60 & 629.36 & $1,179.04$ & 386.44 & 0.49 \\
\hline & Roca Mágica 30\% & 813.87 & 750.82 & $1,406.59$ & 592.72 & 0.73 \\
\hline & Zeolite C $25 \%$ & 759.12 & 387.22 & 725.42 & -33.70 & -0.04 \\
\hline & Zeolite C $30 \%$ & 790.63 & 582.73 & $1,091.70$ & 301.07 & 0.38 \\
\hline \multirow{5}{*}{ Laurel } & Sin Zeolita & 727.53 & 402.75 & 754.51 & 26.98 & 0.04 \\
\hline & Roca Mágica 25\% & 773.72 & 585.65 & $1,097.16$ & 323.44 & 0.42 \\
\hline & Roca Mágica 30\% & 814.03 & 847.14 & $1,587.04$ & 773.01 & 0.95 \\
\hline & Zeolite C $25 \%$ & 772.53 & 577.04 & $1,081.03$ & 308.50 & 0.40 \\
\hline & Zeolite C $30 \%$ & 754.37 & 415.45 & 778.31 & 23.94 & 0.03 \\
\hline \multirow{5}{*}{ Fernánsanchez } & Sin Zeolita & 797.85 & 911.16 & $1,706.97$ & 909.12 & 1.14 \\
\hline & Roca Mágica 25\% & 842.75 & $1,085.66$ & $2,033.87$ & $1,191.12$ & 1.41 \\
\hline & Roca Mágica 30\% & 779.15 & 594.72 & $1,114.15$ & 335.00 & 0.43 \\
\hline & Zeolite C $25 \%$ & 779.66 & 628.67 & $1,177.75$ & 398.09 & 0.51 \\
\hline & Zeolite C $30 \%$ & 800.53 & 749.51 & $1,404.13$ & 603.60 & 0.75 \\
\hline \multirow{5}{*}{ Caoba } & Sin Zeolita & 739.06 & 486.17 & 910.80 & 171.74 & 0.23 \\
\hline & Roca Mágica 25\% & 785.78 & 672.95 & $1,260.71$ & 474.93 & 0.60 \\
\hline & Roca Mágica 30\% & 791.60 & 684.79 & $1,282.89$ & 491.29 & 0.62 \\
\hline & Zeolite C $25 \%$ & 771.51 & 569.68 & $1,067.23$ & 295.72 & 0.38 \\
\hline & Zeolite C $30 \%$ & 761.80 & 447.50 & 838.35 & 76.55 & 0.10 \\
\hline
\end{tabular}




\section{Conclusiones}

Con la combinación fernánsanchez con cacao "CCN-51", se registró una mayor brotación del cacao en la época lluviosa. En la época de seca las asociaciones guayacán con cacao y fernánsanchez con cacao registraron la mayor brotación con el 30\% de ZC y $\mathrm{RM}$, respectivamente.

En los sistemas agroforestales fernánsanchez con cacao y laurel con cacao se registraron las mayores floraciones del cacao. Las asociaciones fernánsanchez con cacao y guayacán con cacao registraron una mayor fructificación con el $30 \%$ de ZC, en la época lluviosa.

Con la asociación fernánsanchez en la época seca se obtuvo el mayor número de mazorcas sanas y producción de cacao por hectárea, y la misma especie con RM al $25 \%$ presentó la mayor rentabilidad. La fertilización con la inclusión de zeolita RM se registró un mayor número de mazorcas sanas y rendimiento de cacao seco. El cacao "CCN-51" presentó su mayor producción en la época seca.

\section{LiteraTURA CITADA}

Barber, S. A. 1995. Soil nutrient bioavailability: a mechanistic approach. 2nd Edn. John Wiley \& Sons, New York, USA, 414 p.

Beer, J., R. Muschler, D. Kass, E. Somarriba. 1998. Shade management in coffee and cacao plantations. Agroforestry Systems 38: 139-164.

Braudeau, J. 1981. El cacao. Ed. Blume. Madrid, ES. $283 \mathrm{p}$.

Concha, J. Y., J. Alegre, V. Pocomucha. 2007. Determinación de las reservas de carbono en la biomasa aérea de sistemas agroforestales de Theobroma cacao L. en el departamento de San Martín, Perú. Ecología Aplicada 6(1, 2): 1-8.

Collazos-García, H. 2010. La aplicación de Zeolita en la producción avícola: Revisión. Revista de Investigación Agraria y Ambiental 1(1): 17-23.

Dahlquist, R.M., M. Whelan, L. Winowiecki, B. Polidoro, S. Candela, C. Harvey, J. Wulfhorst, P. McDaniel, N. Bosque-Pérez. 2007. Incorporating livelihoods in biodiversity conservation: A case study of cacao agroforestry systems in Talamanca, Costa Rica. Biodiversity and Conservation. 16: 2311-2333.

Díaz-Coronel, G.T., F. Sánchez-Mora, L. LlerenaRamos, G. Vásconez-Montúfar. 2009. Empleo de zeolitas naturales en la fertilización y producción del fréjol (Phaseolus vulgaris L.) en la zona de Quevedo. Ciencia y Tecnología 2(1): 1-6.

Enríquez. G. 2004. Cacao orgánico: guía para productores ecuatorianos. INIAP. Quito - Ecuador. 359 p.
Flores, M. A., A. Galvis, T. Hernández, F. León, F. Payán. 2007. Efecto de la adición de zeolita en un andosol sobre el ambiente químico y edáfico y el crecimiento de la avena. Asociación Interciencia 32(10): 692-696.

Isaac, M. E., V. Timmer, S. Quashie-Sam. 2007. Shade tree effects in an 8-year-old cocoa agroforestry system: biomass and nutrient diagnosis of Theobroma cacao by vector analysis. Nutr $\mathrm{Cycl}$ Agroecosyst 78: 155-165.

Jaimez, R. E., W. Franco. 1999. Producción de hojarasca, aporte en nutrientes y descomposición en sistemas agroforestales de cacao y frutales. Agrotrópica 11(1): 1-8.

Jaimez, R. E, W. Tezara, I. Coronel, R. Urich. 2008. Ecofisiología del cacao (Theobroma cacao): $\mathrm{Su}$ manejo en el sistema agroforestal. Sugerencias para su mejoramiento en Venezuela. Revista Forestal Venezolana. 52(2): 253-258.

Jhon, L., G. Del Vallín, G. Dueñas. 1998. Eficiencia de la zeolita como aditivo en la urea en los cultivos de papa y tomate. Instituto de Suelos e Instituto de Cítricos y otros frutales. La Habana - Cuba. 45 p.

Musálem-Santiago, M. Á. 2002. Sistemas agrosilvopastoriles: Una alternativa de desarrollo rural sustentable para el trópico mexicano. Revista Chapingo Serie Ciencias Forestales y del Ambiente 8(2): 91-100.

Orozco, L., M. Villalobos, A. Ortiz. L. Riascos, J. Méndez, V. Sánchez. 2008. Las fincas indígenas bribri y cabécar de Talamanca, Costa Rica. Agroforestería en las Américas. 46:14-20.

Palencia, G., R. Gómez, J. Martín, G. Pérez. 2006. Especies forestales para uso en sistemas agroforestales con cacao. CORPOICA - E.E. La Suiza, Rio Negro, Sder. Bucaramanga, CO. 12p.

Ramesh, K., D. Reddy. 2011. Zeolites and their potential uses in agriculture. Advances in Agronomy 113: 219-241.

Sambuichi, R. H. 2006. Estrutura e dinâmica do componente arbóreo em área de cabruca na região cacaueira do sul da Bahia, Brasil. Acta Botanica Brasilica 20(4): 943-954.

Sánchez-Mora, F., F. Garcés, J. Vera, R. Ramos, F. Troya, T. Díaz. 2011. Cuantificación de enfermedades en mazorcas de cacao (Theobroma cacao L.) en la zona central del Litoral Ecuatoriano. In: Memorias del VIII Simposio Internacional de Recursos Genéticos para América Latina y El Caribe, Quito - Ecuador. Miscelánea No 191 (Resumen expandido).

Scholefield, P. B., M. Sedgley, A. D. McE. 1985. Carbohydrate cycling in relation to shoot growth, floral initiation and development and yield in the 
avocado. Scientia horticulturae 25: 99-110.

Schwendenmann, L., E. Veldkamp, G. Moser, D. Hölscher, M. Köhler, Y. Clough§, I. Anas, G. Djajakiran., S. Erasmi, E. Hertel, D. Leitner, C. Leuschner, B. Michalzik, P. Propastin, A. Tjoa, T. Tscharntke, O. Van Straaten. 2010. Effects of an experimental drought on the functioning of a cacao agroforestry system, Sulawesi, Indonesia. Global Change Biology 16: 1515-1530.

Somarriba, E., J. Beer, R. Muschler. 2001. Research methods for multistrata groforestry systems with coffee and cacao: recommendations from two decades of research at CATIE. Agroforestry Systems 53: 195-203.
Wojtkowski, P. A., C. Jordan, F. Cubbage. 1991. Bioeconomic modeling in agroforestry: a rubbercacao. Agroforestry Systems 14: 163-177.

Zhu, X. G., S. Long, D. Ort. 2008. What is the maximum efficiency with which photosynthesis can convert solar energy into biomass?. Current Opinion in Biotechnology 19: 153-159. 